\title{
Genome-wide identification and characterization of gibberellin metabolic and signal transduction (GA MST) pathway mediating seed and berry development (SBD) in grape (Vitis vinifera L.)
}

Wenran Wang ${ }^{1,2}$, Yunhe Bai', Padmalatha Koilkonda ${ }^{3}$, Le Guan', Yaxian Zhuge ${ }^{1}$, Xicheng Wang ${ }^{4}$, Zhongjie Liu', Haifeng Jia', Chen Wang ${ }^{1 *}$ and Jinggui Fang ${ }^{1 *}$

\begin{abstract}
Background: Grape is highly sensitive to gibberellin (GA), which is crucial during seed and berry development (SBD) either by itself or by interacting with other hormones, such as auxin, Abscisic acid (ABA), and Cytokinin (CK). However, no systematic analysis of GA metabolic and signal transduction (MST) pathway has been undertaken in grapevine.

Results: In this study, total endogenous $\mathrm{GA}_{3}$ content significantly decreased during $\mathrm{SBD}$, and a total of 48 known genes in GA metabolic (GAM; 31) and signal transduction (ST; 17) pathways were identified in this process. In the

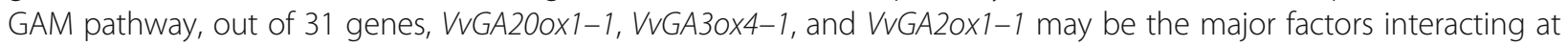
the green-berry stage (GBS) accompanied with higher accumulation rate. GA biosynthesis was greater than GA inactivation at GBS, confirming the importance of seeds in GA synthesis. The visible correlation between endogenous $\mathrm{GA}_{3}$ content and gene expression profiles suggested that the transcriptional regulation of $\mathrm{GA}$ biosynthesis pathway genes was a key mechanism of GA accumulation at the stone-hardening stage (SHS).

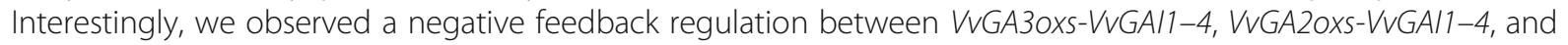
$V_{V G I D 1 B-V V G A / 1-4}$ in maintaining the balance of $\mathrm{GA}_{3}$ content in berries. Moreover, 11 miRNAs may be involved in the modulation of GA MST pathway by mediating their target genes, such as $V_{V} G A 3 O X, V_{V} G I D 1 B$, and $V_{V} G A M Y B$. Many genes in auxin, ABA, and CK MST pathways were further identified and found to have a special pattern in the berry, and the crosstalk between GA and these hormones may modulate the complex process during SBD through the interaction gene network of the multihormone pathway. Lastly, based on the expression characterization of multihormone MST pathway genes, a proposed model of the GA-mediated multihormone regulatory network during SBD was proposed.

(Continued on next page)
\end{abstract}

\footnotetext{
* Correspondence: wangchen@njau.edu.cn; 826507957@qq.com

${ }^{1}$ Nanjing Agricultural University, College of Horticulture, Nanjing 210095, PR

China

Full list of author information is available at the end of the article
}

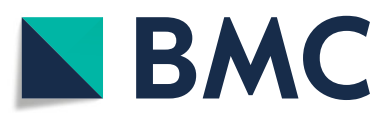

(- The Author(s). 2020 Open Access This article is licensed under a Creative Commons Attribution 4.0 International License, which permits use, sharing, adaptation, distribution and reproduction in any medium or format, as long as you give appropriate credit to the original author(s) and the source, provide a link to the Creative Commons licence, and indicate if changes were made. The images or other third party material in this article are included in the article's Creative Commons licence, unless indicated otherwise in a credit line to the material. If material is not included in the article's Creative Commons licence and your intended use is not permitted by statutory regulation or exceeds the permitted use, you will need to obtain permission directly from the copyright holder. To view a copy of this licence, visit http://creativecommons.org/licenses/by/4.0/. The Creative Commons Public Domain Dedication waiver (http://creativecommons.org/publicdomain/zero/1.0/) applies to the data made available in this article, unless otherwise stated in a credit line to the data. 
(Continued from previous page)

Conclusions: Our results provided novel insights into GA-mediated regulatory networks during SBD in grape. The complexity of GA-mediated multihormone ST in SBD was also elucidated, thereby providing valuable information for future functional characterizations of specific genes in grape.

Keywords: Grape berry and seed development, GA MST pathway, Multihormone MST pathway,

\section{Background}

Grape (Vitis vinifera L.), which is a perennial fruit crop cultivated globally, is economically important playing a major role for human health served as fresh food, dried food, and beverages and valued for its medicinal importance. The seed and berry development (SBD) is one of the extremely important phases during its life cycle. Grape is highly sensitive to gibberellin (GA), which is one of the essentially significant hormones during SBD. The molecular and genetic analysis in previous studies revealed that GA signal is involved in various stages of berry growth and development [1], such as promoting berry expansion, significantly affecting fruit set, coloring, and development, and ripening of berries. Several studies have demonstrated the exogenous prebloom application of $\mathrm{GA}_{3}$-induced seed abortion in the 'Zuijinxiang' and 'Red Globe' seeded cultivars [2, 3]. Furthermore, GA can increase fruit sink strength [4]; thus, GA can indirectly affect fruit development through affecting energy supplement. During the past two decades, a set of genes involved in GA signal pathway have been identified, and the biological functions of key genes have been extensively characterized in banana [5] and apple [6]. The molecular interaction network of GA signal pathway in regulating $\mathrm{SBD}$ at the whole wide-transcriptome level is still unclear in grape.

The control of DELLA stability is a key factor for the GA response and represents a major entry point importance for other phytohormones, such as IAA [7] and ABA [8], affecting plant growth and development. Therefore, we supposed that a network exists between DELLA proteins that mediated GA metabolic and signal transduction (MST) pathways and a cross talk with multihormone MST pathway during SBD. A complete understanding of the GA, ABA, auxin, and CK MST pathways is fundamental for elucidating the mechanisms during grape SBD. With the release of the grape genome sequence (http://genomes.cribi.unipd.it/DATA/V2/V2/) and the increasing affordability of high throughput analysis tools, a better opportunity is present to study the genes related to MST pathways in grape systematically.

The morphological and physiological variation during development of berries obviously implies the expression level variation of many genes, including genes in GA MST pathway. On the basis of the RNA-seq data from four grape berry developmental stages, we identified and characterized the genes involved in GA, auxin, CK, and ABA MST pathways in grape berries and detected their spatio-temporal expression profiles during SBD, together with the accumulation variation of $\mathrm{GA}_{3}$, IAA, $\mathrm{ZR}$, and ABA contents along with the berry development, to gain insight into the signal regulatory networks of SBD at the whole wide-transcriptome level. A complex regulatory network of GA-mediating SBD with other hormone cooperative regulations in grape was developed. Our research outcome could enhance understanding and provide valuable information for further elucidation of molecular mechanisms and underlying interactions between GA and ABA, Auxin, CK regulation during SBD.

\section{Results \\ Morphological, physiological, and biochemical variations during SBD}

Figure 1a shows that grape berries continued growing from green-berry stage (GBS) until ripening period, whereas grape seeds developed maturity and hardened by SHS. The developmental curve and average weight of berries and seed showed a rapid increase from GBS to SHS, whereas they maintained stability during berry-veraison stage (BVS) and berry-ripening stage (BRS) (Fig. 1b and c). During initial berry growth and GBS, berry size increased along a sigmoidal growth curve due to cell division and expansion. SHS is defined as a lag phase in which cell expansion ceases, seeds harden, embryo rapidly develops, and sugars begin to accumulate. BVS marks the beginning of the BRS in which berries undergo a second period of sigmoidal growth due to additional mesocarp cell expansion, accumulation of anthocyanin, glucose, and fructose, and a decline in organic acid accumulation.

\section{Contents of endogenous $\mathrm{GA}_{3}, \mathrm{IAA}, \mathrm{ZR}$, and $\mathrm{ABA}$}

The levels of endogenous hormones $\mathrm{GA}_{3}$, IAA, and $\mathrm{ZR}$ during berry development both increased and decreased, whereas the levels of ABA decreased. The highest levels of $\mathrm{GA}_{3}$, IAA, and $\mathrm{ZR}$ were observed at SHS, whereas ABA was at GBS. The total amount of $\mathrm{GA}_{3}$, IAA, and ZR (expressed as $\mathrm{ng} / \mathrm{g} \mathrm{fw}$ ) progressively increased from $1.90,21.38$, and $5.81 \mathrm{ng} / \mathrm{g}$ fw at GBS to $14.24,99.62$, and19.28 ng/g fw at SHS, and decreased from to 7.00, 78.93, and $17.84 \mathrm{ng} / \mathrm{g}$ fw at BRS (Fig. 1d). ABA was detected at $119.73 \mathrm{ng} / \mathrm{g} \mathrm{fw}$ at GBS to $41.67 \mathrm{ng} / \mathrm{g}$ fw in ripe berry. 


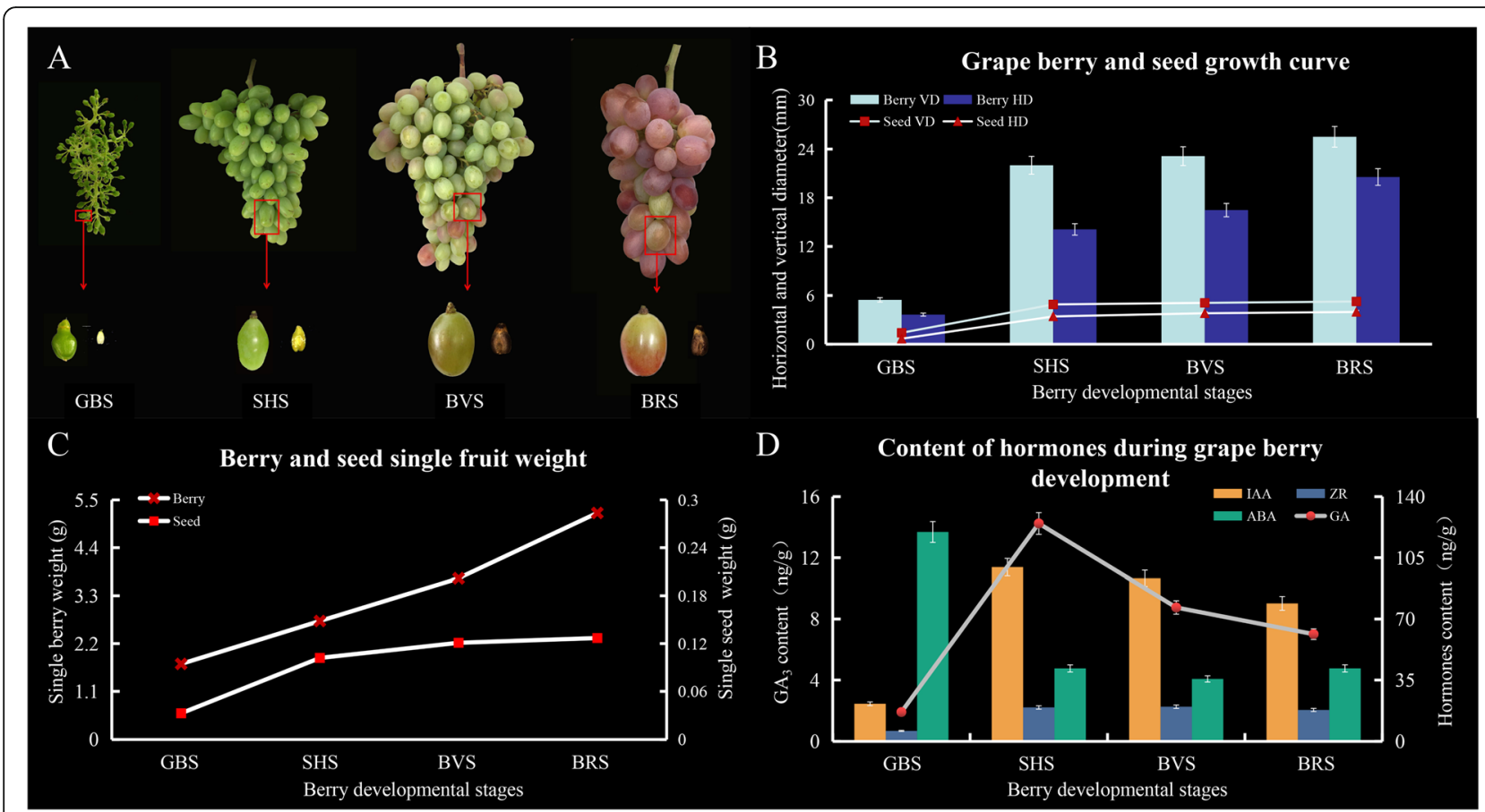

Fig. 1 Growth and Variation in berry size during development of berries at four stages (GBS, SHS, BVS and BRS). a Color and size variation in clusters, berries and seeds at GBS, SHS, BVS and BRS. b Variation in average single seed and berry weights at four developmental stages. c Variation in average length and width of single seed and berry during four developmental stages. $\mathbf{d}$ Endogenous levels of GA 3 , IAA, ZR and $A B A$ during four stages of development of berries

\section{Identification of genes in GA MST pathway}

In grape, a total of 48 non-redundant GA MST-related genes were identified in this study. Each of them was named based on the enzymatic reaction and referred to Arabidopsis thaliana GA MST pathway. Among these 48 GA MST-related genes, 31 and 17 genes were involved in GAM and ST, respectively. These genes belonged to nine different gene families, and all of them were identified as single gene copy. Two genes showed no change in their expression levels during SBD, including $V v G A 200 x 1-2$ and $V \nu G A 20 o x 2-1$, and 2 transcripts were observed as upregulated or downregulated slightly. About 44 transcripts were considered as significantly differentially expressed genes (Tables 1 and 2).

\section{In silico characterization of genes in GA MST pathway during berry development \\ Chromosomal distribution and collinearity of genes}

A total of 48 genes in GA MST pathway could be assigned to 18 out of 19 grape chromosomes (except for Chr 2), and uneven distribution of genes across the chromosomes was observed. Chr9 harbored most of the genes (8), followed by Chr4 (6), Chr7 (4), Chr19 (4), Chr17 (3), and the remaining Chrs with 1-2. The results revealed that the genes, such as $V \nu G A 20 o x s$ and $V \nu G A 3 o x s$, in the GA biosynthesis pathway tended to localize on the same Chrs, that is, on Chr 4 and 9, whereas $V \nu G A 2 o x s$ in GA inactive pathway were localized on the same Chrs, that is, Chr1, 7, and 19, also the genes in GA signal pathway such as $V v G I D 1 B(\mathrm{Chr} 7)$, VvGAIs (Chr1, 7, 14, and 17), and VvRGL1 (Chr9). These results indicated that similar functional genes may possess certain conservation in their locus in grape genome (Fig. 2a).

Furthermore, 12 genes (i.e., $V v G A 3 o x 4-1 / V \nu G A 3 o x 1-2$, $V \nu G A 3 o x 1-4 / V v G A 20 o x 3-1 / V v G A 20 o x 1-3 / V v G A 3 o x 1-$ 3/VvGA2ox2-3/VvGA20ox3-2/VvGA3ox1-1/VvGA3ox3, and $V \nu G A 2 o x 1-1 / V \nu G A 2 o x 1-3)$ were clustered into three tandem duplication event regions on grape Chr4, 9, and 19 (Fig. 2b). Besides the tandem duplication events, three segregation duplication events (i.e., $V v G A 20 o x 1-B 1 /$ $V \nu G A 3 o x 4-3, \quad V v G A 20 o x 1-B 2 / V v G A 3 o x 4-3, \quad$ and $V \nu G A 3 o x 1-4 / V \nu G A 3 \circ \times 1-3)$ were also identified (Fig. 2b), indicating that some grape genes in GA MST pathway were possibly generated by gene duplication. Moreover, the segregation duplication events can also provide a reference for the GA MST gene evolutionary relationship and functional prediction.

\section{Phylogenetic tree and exon-intron organization of genes in GA MST pathway}

The phylogenetic tree classified the proteins coded by genes in the GA MST pathway into two groups, G1 and G2 (Fig. 2c). All genes in GA metabolism pathway were 
Table 1 GA Metabolic pathway genes in grape berry

\begin{tabular}{|c|c|c|c|c|c|c|c|}
\hline Name & Accession no. & Family & Chrom & Gene start & Gene end & Gene length & Gene strand \\
\hline VVGA20ox1-B1 & VIT_215s0046g02550 & 2OG-Fe (II) oxygenase & chr15 & $19,336,280$ & $19,337,354$ & 987 & + \\
\hline VVGA20ox1-B2 & VIT_216s0050g00640 & 2OG-Fe (II) oxygenase & chr16 & $17,592,866$ & $17,594,861$ & 984 & - \\
\hline VVGA200x1-1 & VIT_203s0063g01150 & 2OG-Fe (II) oxygenase & chr3 & $4,642,814$ & $4,644,285$ & 1029 & - \\
\hline VVGA20ox1-2 & VIT_202s0234g00010 & 2OG-Fe (II) oxygenase & chr2 & 11,691 & 13,858 & 1074 & - \\
\hline VVGA20ox1-3 & VIT_209s0002g05290 & 2OG-Fe (II) oxygenase & chr9 & $5,011,954$ & $5,013,413$ & 936 & - \\
\hline VVGA20ox2-1 & VIT_218s0001g01390 & 2OG-Fe (II) oxygenase & chr18 & $1,982,611$ & $1,985,550$ & 1137 & - \\
\hline VVGA20ox2-2 & VIT_215s0048g01320 & 2OG-Fe (II) oxygenase & chr15 & $15,453,736$ & $15,455,160$ & 1155 & + \\
\hline VVGA20ox2-3 & VIT_204s0044g01520 & 2OG-Fe (II) oxygenase & chr4 & $23,110,636$ & $23,112,150$ & 1128 & - \\
\hline VVGA20ox2-4 & VIT_204s0044g01650 & 2OG-Fe (II) oxygenase & chr4 & $23,382,366$ & $23,384,136$ & 1134 & - \\
\hline VVGA200x3-1 & VIT_209s0002g05280 & 2OG-Fe (II) oxygenase & chr9 & $5,008,806$ & $5,010,211$ & 945 & - \\
\hline VVGA20ox3-2 & VIT_209s0002g05320 & 2OG-Fe (II) oxygenase & chr9 & $5,054,916$ & $5,056,221$ & 942 & - \\
\hline VVGA200X & VIT_216s0022g02310 & 2OG-Fe (II) oxygenase & chr16 & $14,861,534$ & $14,863,137$ & 1149 & - \\
\hline VVGA3OX1-1 & VIT_209s0002g05340 & 2OG-Fe (II) oxygenase & chr9 & $5,075,023$ & $5,077,440$ & 951 & - \\
\hline$V V G A 30 \times 1-2$ & VIT_204s0008g04940 & 2OG-Fe (II) oxygenase & chr4 & $4,431,414$ & $4,432,859$ & 1068 & - \\
\hline VVGA3OX1-3 & VIT_209s0002g05300 & 2OG-Fe (II) oxygenase & chr9 & $5,037,560$ & $5,040,878$ & 1080 & - \\
\hline VVGA3OX1-4 & VIT_209s0002g05270 & 2OG-Fe (II) oxygenase & chr9 & $4,993,458$ & 4,995,066 & 1098 & + \\
\hline VVGA3OX3 & VIT_209s0002g05350 & 2OG-Fe (II) oxygenase & chr9 & $5,095,320$ & $5,096,737$ & 954 & - \\
\hline VVGA3OX4-1 & VIT_204s0008g04920 & 2OG-Fe (II) oxygenase & chr4 & $4,415,690$ & $4,418,033$ & 951 & + \\
\hline VVGA3ox4-2 & VIT_204s0044g02010 & 2OG-Fe (II) oxygenase & chr4 & $23,794,890$ & $23,796,653$ & 897 & - \\
\hline VVGA3OX4-3 & VIT_202s0025g03440 & 2OG-Fe (II) oxygenase & chr2 & $2,939,781$ & $2,941,071$ & 966 & + \\
\hline VVGA2OX1-1 & VIT_219s0140g00120 & 2OG-Fe (II) oxygenase & chr19 & $15,497,747$ & $15,499,547$ & 972 & + \\
\hline VVGA2OX1-2 & VIT_213s0067g01150 & 20G-Fe (II) oxygenase & chr13 & 635,013 & 637,333 & 1149 & + \\
\hline VVGA2OX1-3 & VIT_219s0140g00140 & 2OG-Fe (II) oxygenase & chr19 & $15,603,314$ & $15,604,866$ & 999 & + \\
\hline VVGA2OX2-1 & VIT_205s0077g00520 & 2OG-Fe (II) oxygenase & chr5 & 343,963 & 346,779 & 1020 & - \\
\hline$V V G A 2 O \times 2-2$ & VIT_207s0005g01920 & 2OG-Fe (II) oxygenase & chr7 & $4,377,229$ & $4,379,446$ & 1002 & + \\
\hline VVGA2OX2-3 & VIT_209s0002g05310 & 2OG-Fe (II) oxygenase & chr9 & $5,051,513$ & $5,052,701$ & 813 & - \\
\hline VVGA2OX8-1 & VIT_201s0010g01650 & 2OG-Fe (II) oxygenase & chr1 & $17,965,019$ & $17,969,123$ & 1092 & - \\
\hline VVGA2OX8-2 & VIT_219s0177g00030 & 2OG-Fe (II) oxygenase & chr19 & $5,864,515$ & $5,868,916$ & 1104 & - \\
\hline VVGA2OX8-3 & VIT_210s0116g00410 & 2OG-Fe (II) oxygenase & chr10 & 190,070 & 195,160 & 1035 & - \\
\hline VVGA2OX8-4 & VIT_206s0004g06790 & 2OG-Fe (II) oxygenase & chr6 & $7,476,208$ & $7,478,569$ & 1014 & + \\
\hline VVGA2OX & VIT_210s0003g03490 & 2OG-Fe (II) oxygenase & chr10 & $5,846,605$ & $5,848,332$ & 1002 & + \\
\hline
\end{tabular}

Table 2 GA Signal transduction pathway genes in grape berry

\begin{tabular}{llllllll}
\hline Name & Accession no. & Family & Chrom & Gene start & Gene end & Gene length & Gene strand \\
\hline VVGID2-1 & VIT_207s0129g01000 & F-box & chr7 & $16,134,715$ & $16,135,491$ & 555 & + \\
VVGID2-2 & VIT_218s0001g09700 & F-box & chr18 & $8,077,244$ & $8,078,167$ & 555 & + \\
VVGID1B & VIT_207s0104g00930 & GID1 & chr7 & $2,061,408$ & $2,063,888$ & 1035 & - \\
VVGAl1-1 & VIT_214s0068g01610 & GRAS & chr14 & $25,316,395$ & $25,318,488$ & 2007 & - \\
VVGAl1-2 & VIT_217s0000g10300 & GRAS & chr17 & $12,556,730$ & $12,558,304$ & 1560 & - \\
VVGAl1-3 & VIT_207s0005g01500 & GRAS & chr7 & $4,026,350$ & $4,028,863$ & 1815 & - \\
VVGAl1-4 & VIT_214s0006g00640 & GRAS & chr14 & $14,806,872$ & $14,809,122$ & 1710 & + \\
VVGAl1-5 & VIT_201s0011g05260 & GRAS & chr1 & $4,895,037$ & $4,897,415$ & 1773 & - \\
VVRGL1 & VIT_219s0085g00540 & GRAS & chr19 & $22,922,082$ & $22,923,674$ & 1593 & + \\
VVSLR1 & VIT_211s0016g04630 & GRAS & chr11 & $3,959,481$ & $3,961,177$ & 1599 & + \\
\hline
\end{tabular}




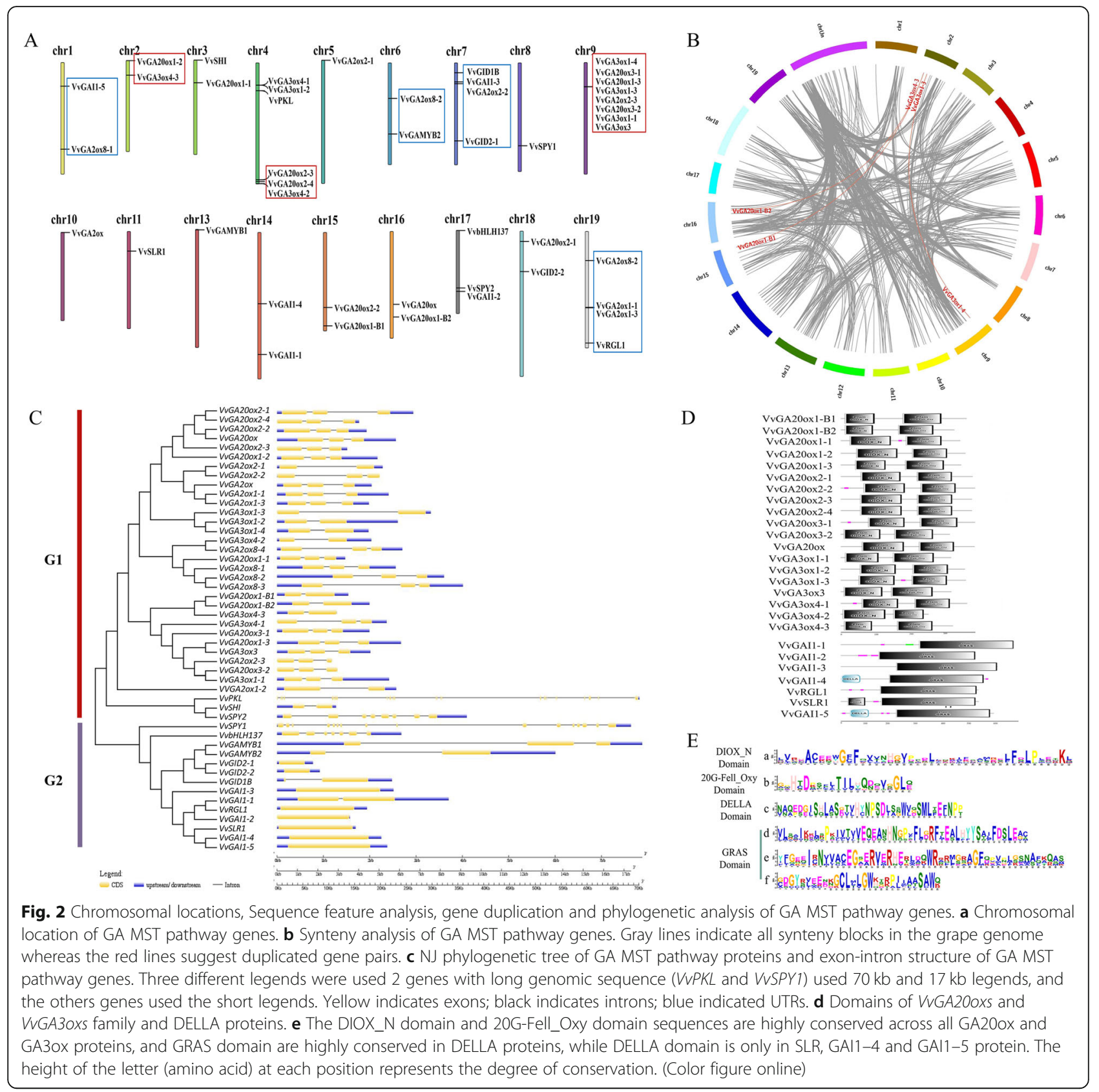

clustered into G1, whereas except for $V v P K L, V v S H I$, and $V v S P Y 2$, the remaining genes were clustered in GA ST pathway into G2.

Unlike their locations in Chrs, $V \nu G A 20 o x$ s was closer to $V \nu G A 2 o x s$ than $V \nu G A 3 o x s$. Further analysis of the exon-intron structure on the genes in GA MST pathway showed that similar to their phylogenetic tree, the exon numbers of the majority of genes in G1 were 3 or 2, whereas those in G2 were 1 (Fig. 2d). However, high variation was observed in the number of exons and introns in all genes in GA MST pathway. Eight genes (i.e., VvGID2-1, VvGID2-2, VvGAI1-3, VvRGL1, VvGAI1-2,
$V v S L R 1, V v G A I 1-4$, and $V v G A I 1-5)$ had no intron in the grape genome; whereas $V v P K L$ was the longest gene with $70 \mathrm{~kb}$ genomic sequence in GA MST pathway and contained the most exons (29) in this study, followed by $V v S P Y 1$ with 16 exons, VvSPY2 and VvbHLH137 with 8/ 7 , respectively, whereas the remaining 42 genes all had 1-3 exons (Fig. 2d).

\section{Features of conserved domains and motifs}

The DIOX_N domain and 20G-Fell_Oxy domain are common domains of $20 V v G A 20 o x s$ and VvGA3oxs. On the basis of the same domains, we identified the highly 
conserved motif of DIOX_N domain (Fig. 2. F-a) and 20G-Fell_Oxy domain (Fig. 2. F-b). Additionally, $V v G A 20 o x s$ and $V v G A 3 o x s$ were localized on the same Chrs. Similar to $V v G A 20 o x s$ and $V v G A 3 o x s$, three highly conserved motifs of GRAS domain were identified in seven DELLA proteins. DELLA domain was found in $V v G A I 1-4, V v S L R 1$, and $V v G A I 1-5$. Furthermore, they clustered into the same branch of phylogenetic tree, suggesting they may have similar function.

\section{Expression analysis of genes in GA MST pathway Biosynthesis pathway}

In this study, 20 genes, including 12 GA20ox and 8 GA3ox, were involved in GA biosynthesis pathway (Fig. 3a and b; Additional file 1: Table S1), are particularly important for control of bioactive GA levels. Among $12 V v G A 200 x s, V v G A 20 o x 1-1$ showed the highest expression levels at GBS with significant expression levels in all four stages. VvGA20ox1-B1, VvGA20ox1-B2, $V v G A 200 \times 1-2$, and $V v G A 20 o x 2-1$ showed no expression during SBD. Some members of VvGA20oxs family showed significantly lower expression in $V v G A 20 o x 1-3$, $V v G A 20 o \times 2-2, \quad V v G A 20 o \times 2-4, \quad V v G A 2 o \times 3-1, \quad$ and $V v G A 20 o x$. Similarly, among the 8 members of another significant enzyme GA3ox family, the expression of $V v G A 3 o x 4-1$ was higher than that of $V v G A 3 o x 4-2$ and $V v G A 3 o x 1-4$. They showed the highest expression at GBS of berries, and its expression significantly decreased from GBS to BRS down to the lowest level, whereas other members only had slight or no expression during SBD.

\section{Inactivation pathway}

$V v G A 2 o x s$ is an enzyme used in GA inactivation pathway. Here, $11 V v G A 2 o x$ genes were identified in berries. $V \nu G A 20 x 1-1$, of which $V v G A 20 \times 1-1, \quad V v G A 2 o x 2-1$,

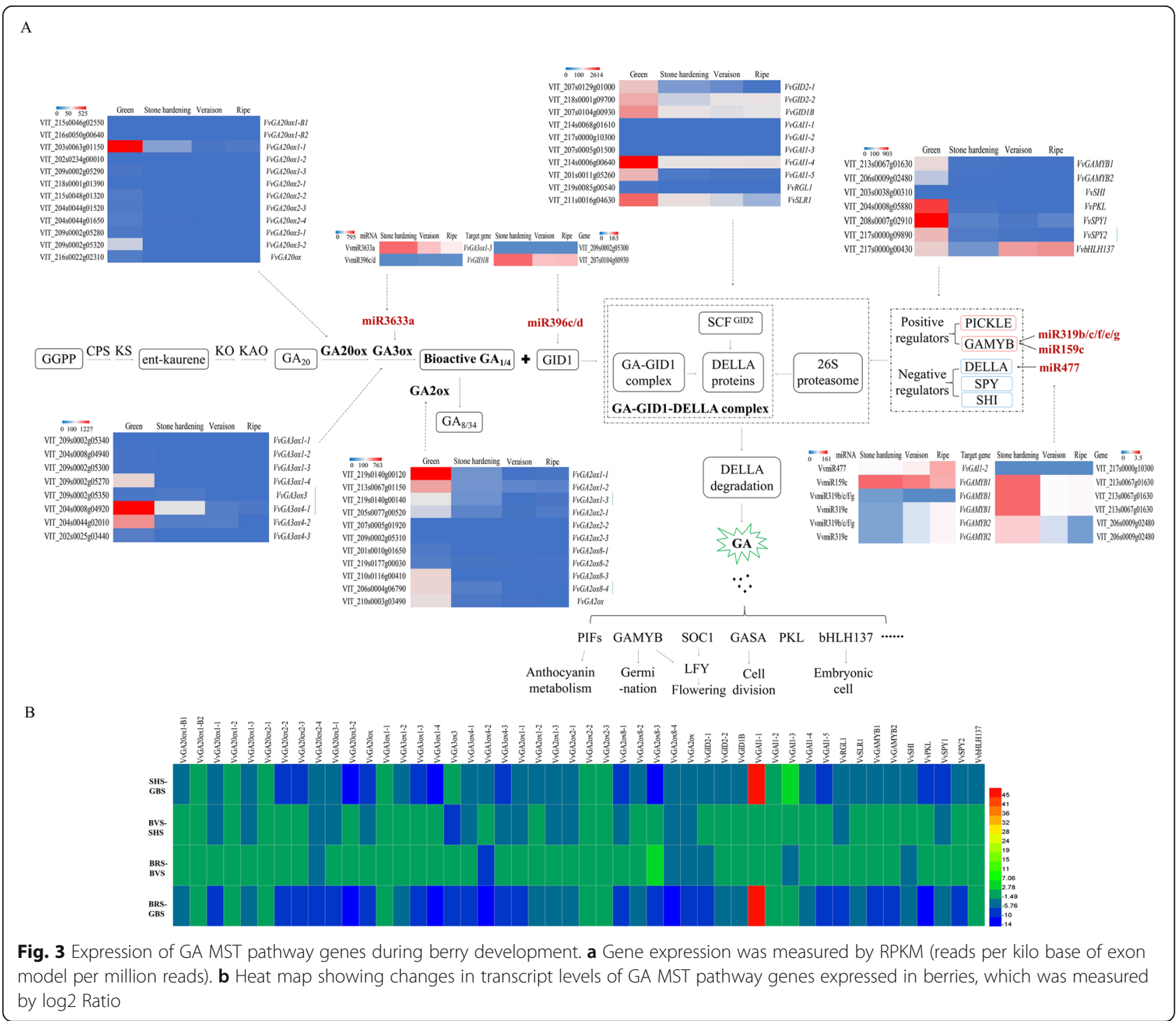


$V v G A 20 x 8-3, V v G A 20 x 8-4$, and $V v G A 2 o x$, exhibited the highest expression and showed significant variation during the four stages of berry development (Fig. 3a and b; Additional file 1: Table S1). They had the highest expression at GBS. Nevertheless, $V \nu G A 2 o x 2-2$, $V \nu G A 2 o x 2-3, V \nu G A 2 o x 8-1$, and $V \nu G A 2 o x 8-2$ were almost not expressed or changed in the four stages of berry development.

\section{Signal transduction pathway}

One $V v G I D 1 B$ gene (GA receptor) was identified and had the highest and lowest expression levels at GBS and BVS, respectively (Fig. 3a). We identified the two members of GID2 family in grape berries that exhibited the expression peak at GBS. VvGID2-1 gradually decreased from the SHS to BRS, whereas $V v G I D 2-2$ exhibited the lowest expression at SHS. We identified seven genes of DELLA family including $V \nu G A I 1-1, \quad V \nu G A I 1-2$, $V \nu G A I 1-3, \quad V v G A I 1-4, \quad V v G A I 1-5, \quad V v R G L 1, \quad$ and $V v S L R 1$. $V v G A I 1-4$ had the highest expression, followed by $V v S L R 1$ and $V v G A I 1-5$, and the remaining genes had lower expressions through grape berry developmental process; $V v G A I 1-4, V \nu S L R 1$, and $V \nu G A I 1-5$ predominantly expressed especially at GBS (Fig. 3a and b; Additional file 1: Table S1).

\section{Regulatory factors}

We revealed that two positive action components $V v G A$ $M Y B$ and $V v P K L$ were primarily expressed at GBS in berries, whereas another four negative action components $V v S P Y 1, V v S P Y 2$, and $V v b H L H 137$ were also detected in berries at four stages, and only $V v S H I$ possessed low expression levels throughout the four stages in berries (Fig. 3a and b; Additional file 1: Table $\mathrm{S} 1)$. A total of $10 \mathrm{VvmiRNAs,} \mathrm{such} \mathrm{as} V v G A 3 o x s$ for VvmiR3633a, $V v G I B 1 B$ for VvmiR396c/d, $V v G A I 1-2$ for VvmiR477, $V v G A M Y B 1$ for VvmiR159c and $\mathrm{VvmiR} 319 \mathrm{~b} / \mathrm{c} / \mathrm{e} / \mathrm{f} / \mathrm{g}$, and $V \nu G A M Y B 2$ for $\mathrm{VvmiR} 319 \mathrm{~b} / \mathrm{c} /$ $\mathrm{e} / \mathrm{f} / \mathrm{g}$, may be involved in the modulation of GA signal pathway by mediating their target genes. These VvmiRNAs and their target genes exhibited the opposite expression trends in berries, indicating that these VvmiRNAs possessed negative regulatory roles on these potential target genes during SBD (Fig. 3a and b; Additional file 1: Table S1). Moreover, among these VvmiRNAs, VvmiR3633a had the highest expression in SHS berries, whereas the remaining ones were slightly expressed in berries. The target gene $V v G I D 1 B$ for VvmiR396c/d exhibited the highest expression also at SHS, implying that VvmiR3633a may repress GA biosynthesis at SHS by modulating the expression level of $V \nu G A 3 o x 1-3$. Given that the VvmiR396c/d had low expression level, resulting into the high expression of $V \nu G I D 1 B$ may strengthen the GA signal receptivity.

\section{Expression characterization of genes in multihormone MST pathways}

From our dataset, we also identified the 62, 36, and 73 genes in auxin, $\mathrm{CK}$, and ABA MST pathway, respectively. $V v Y U C 10-4, V v T A R 4-1, V v A U X, V v A R F 2-2$, and $V v S A U R 2$ of auxin MST pathway, $V v N C E D 1$ and $V \nu P P 2 C 49$ of ABA MST pathway, and $V \nu C K X 5$ of CK MST pathway exhibited higher expression levels than other members of their corresponding gene families (Fig. 4; Additional file 2: Table S2).

\section{Auxin MST pathway}

Both VvYUC10-4 and VvTAR4-1 key enzymes in endogenous auxin biosynthesis showed the increased expression peak at GBS and dramatically decreased at BVS. $V v A R F 2-2$, which is a transcriptional regulator repressing a series of auxin response, exhibited the highest expression gene of 18 ARF gene members. Most of them displayed the highest expression at GBS and then dramatically decreased until BVS of berries. Similar to $V v A R F s$, AUX/IAA protein was also abundant at GBS. Another important element in auxin MST pathway is $V v S A U R$, and its transcription is dependent on the level of active auxin. Different from $V v A R F s$ and AUX/IAA proteins, VVSAUR2 showed high transcription levels through berry development process.

\section{ABA MST pathway}

$V v N C E D 1$ and $V v N E C D 2-1$ genes, which are ratelimiting enzymes for ABA biosynthesis, are highly expressed at GBS. In addition, seven $V v C Y P 707 A s$ were identified in this work for ABA metabolism, and five of them were abundant at GBS. Five ABA receptors were found, but only $V v P Y L 9$ was highly expressed in grape berry and primarily at GBS. Then, $51 \quad V v P P 2 C$ gene members were identified from our database. $V v P P 2 C 25-$ 1 and $V v P P 2 C 49$ were highly expressed than others, and 37 members of PP2C gene family expressed at GBS as well. $V v A B I 5 s$ is primarily expressed at GBS and is higher than other stages during SBD.

\section{CK MST pathway}

In CK MST pathway, compared with the other six members of CKX family, $V \nu C K X 5$ displayed the highest expression levels in berry. A similar trend for $V \nu C K X 3-2$, $V \nu C K X 5$, and $V \nu C K X 7$ was detected at GBS and then dramatically decreased until BRS. Only VvLOG1-2 showed highest levels among the 10 LOG members at GBS. $V \nu C K X s$ was highly expressed than $V \nu L O G s$ at GBS, so we presume that cytokinin may primarily be accumulated during SHS, BVS, and BRS. These results are inconsistent with the variation of endogenous $\mathrm{CK}$ content during SBD (Fig. 1d). 

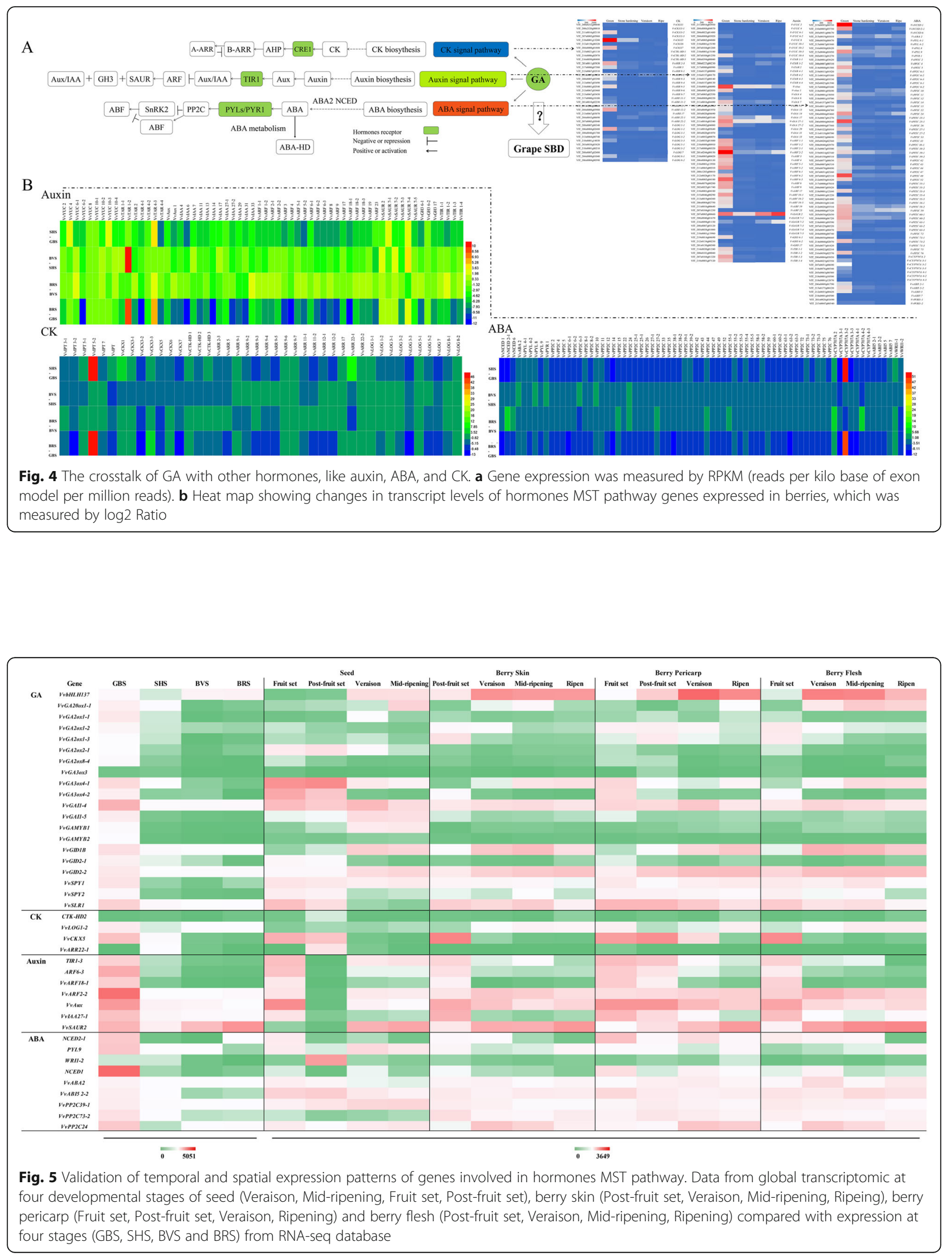
Organ-specific expression pattern analysis of genes in hormone MST pathway at the global transcriptome level of grape berries

To evaluate the validity of our sequencing results, based on the data from $V$. vinifera $\mathrm{cv}$. Corvina global gene expression atlas from the GEO DataSets (GSE36128), which comprising 54 grape tissues, organs or developmental stages [9]. The 64 gene tissue-specific expression patterns during seed, berry skin, berry pericarp, and berry flesh development were identified in this work (Fig. 5; Additional file 3: Table S3). Figure 5 shows that $8,1,3$, and 2 gene expression patterns in seed, berry skin, pericarp, and flesh were similar to our data, respectively. Additionally, 50 genes displayed the same trend in at least two tissues from our database, indicating that multitissues regulated these gene expression. We found genes expressed differently in the same period of different tissues due to their function in various developmental process. For example, VvGA20ox1-1 and $V v G A 3 o x 4-1$ were detected low in veraison and ripe berry in our data, whereas both were highly expressed at veraison and ripe period in berry skin and flesh from the GEO DataSets. It supposed that accumulation of anthocyanin and glucose and fructose are relative to GA, thus two genes highly expressed for GA biosynthesis at two periods.

\section{Data analysis of GA MST pathway genes between RT- qPCR and Illumina RNA-seq}

To validate expression patterns obtained from RNA-Seq, six genes (i.e., $V v G A 200 x 1-1, V v G A 3 o x 4-1, V v G A 2 o x 1-$ 1, $V v G I D 1 B, V \nu G A I 1-4$, and $V \nu S L R 1)$ that were the core genes in GA MST pathway and highly expressed in green berry were selected to validate by using RT-qPCR analysis (Fig. 6). The expression profiles of RT-qPCR were a similar tendency with RNA-Seq results, indicating that the data from RNA-Seq could represent genes relative expression levels at different development stage in grape berry. The relative expression levels of $V v G A 20 \circ x 1-1, V v G A 3 o x 4-1, V v G A 2 o x 1-1, V v G I D 1 B$, $V v G A I 1-4$, and $V v S L R 1$ were highly detected at GBS and dramatically decreased at SHS from Illumina sequencing. Data exhibited that the six genes showed the highest expression at 10 days after anthesis (DAA) or 20 DAA in RT-qPCR results, which were approximately consistent with Illumina sequencing. This difference may be caused by different species with slightly different time points during the development process.

\section{Discussion}

According to previous reports, GA regulatory networks are involved in organogenesis and development of fruit in tomato [10] and strawberry [11]. Moreover, genes involved in GA MST pathway are associated with fruit development in pepper [12], pea [13], tomato [14], and Arabidopsis [15]. Few studies [16] have been conducted on the GA MST and GA-mediated multihormone MST pathway mechanisms in grape. Hitherto, no reports are available on the identification and comparative profiling of GA mediated pathway and multihormones like auxin, $\mathrm{ABA}$, and $\mathrm{CK}$ mediated pathway genes during $\mathrm{SBD}$ in grape.

\section{Genes in GA MST pathway during SBD}

Gene duplication events are defined as either tandem or segmental duplication, which have been identified as two of the main causes for genomic rearrangements and expansions [17]. Holub [18] reported that a tandem duplication event involves three or more copies in chromosomal regions of less than $200 \mathrm{~kb}$. Three tandem duplication event regions on Chr4, 9, and 19 were found in our present study. Moreover, three segregation

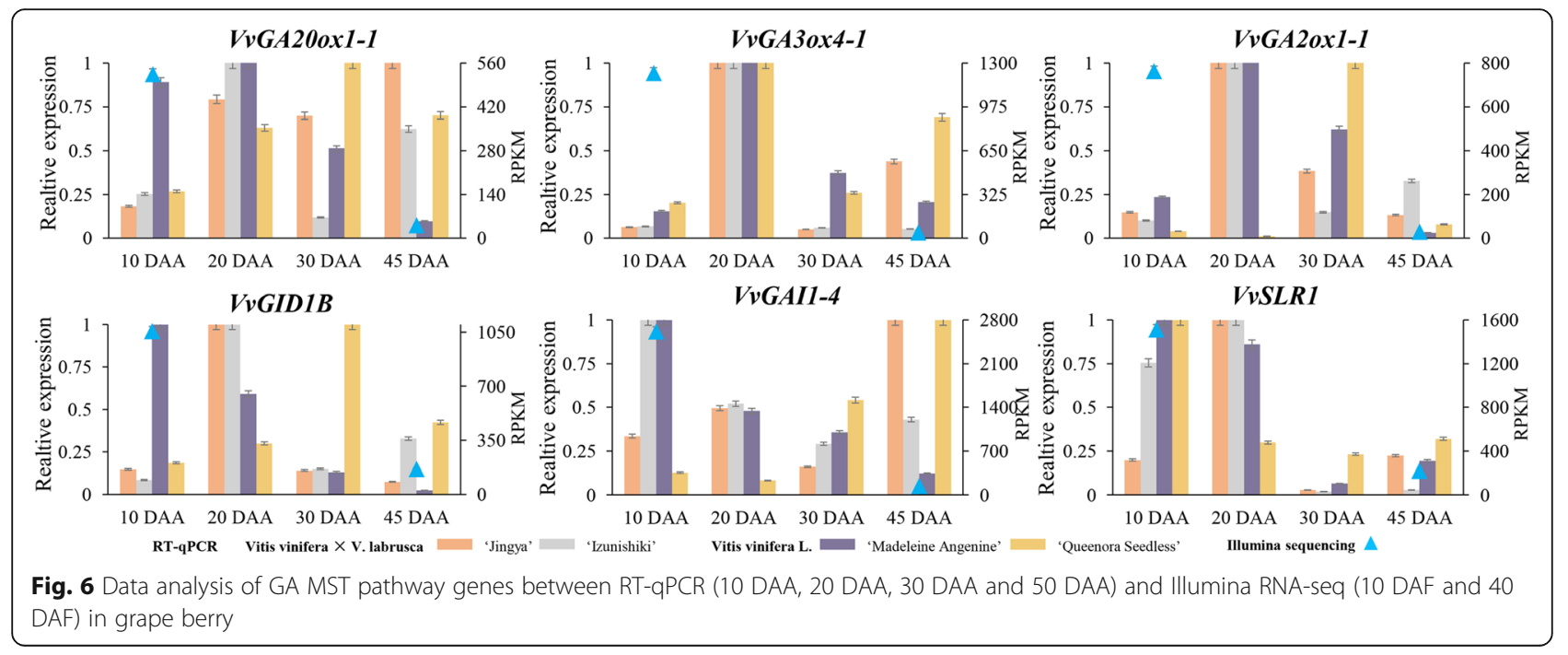


duplication events were also identified, indicating that some GA biosynthesis genes (i.e., VvGA3oxs and $V v G A 200 x s)$ were possibly generated by gene duplication. Overall, the collinearity events can also support the GA biosynthesis gene evolutionary relationship and functional prediction. Based on the phylogenetic tree, we found that the more similar the functions of genes are, the closer they are in relationship. The closer evolutionary relationship may explain the highly conserved motif and large variation in the structures of genes in GA MST pathway, indicating that the grape genome changed significantly during its extensive evolutionary processes.

$V \nu G A 3 o x s$ and $V v G A 20 o x s$ are particularly important in controlling bioactive GA levels [19], and $V \nu G A 2 o x s$ modulate the endogenous GA balance by GA inactivation. In the present study, $V v G A 20 o x s, V v G A 3 o x s$ and $V \nu$ GA2oxs were multicopy genes and exhibited temporal-specific expression pattern in berries, which agree with the studies on rice [20] and Arabidopsis [21]. Field observations and few physiological studies [22] point out that grape SBD are strictly correlated, and seeds are generally a metabolic center of phytohormones [23]. From our studies, grape seeds are one of the organs for endogenous GA biosynthesis, whereas GBS and SHS are important phases for grape SBD. Expression analysis of GA genes indicated that $V v G A 20 o x 1-1$ and $V v G A 3 o x 4-1$ may be key factors in endogenous GA biosynthesis during SBD, especially at GBS. Combined with high expression level of $V v G A 2 o x 1-1$ at GBS, it may be the key factor in modulating the endogenous GA balance by GA inactivation at GBS. Simultaneously, GBS and SHS are the crucial phases for endogenous GA metabolism process, consistent with endogenous GA contents, which increase from GBS to SHS in berries and reach the expression peak at SHS. This phenomenon may be derived from the fact that seeds are the important sites for endogenous GA biosynthesis, whereas the period from GBS to SHS is significant for the development of seeds.

DELLA proteins, which are GA signal pathway receptors, are the core acting elements of GA MST pathway [24]. Our study showed that GBS is a key stage of responding to GA and GA signal transduction during SBD. $V v G A I 1-4$ and $V v S L R 1$ may also be crucial core acting factors of GA signal transduction at GBS. GID1B, which is a GA receptor, plays a key role in switching on GA signal transduction. Given that $V v G I D 1 B$ exhibits high expression at GBS, GBS may be the active stage for strong response to GA signal. Although previous evidence showed that two putative GA receptors increased during grape berry development [25], this discrepancy may be caused by different grape varieties used in the two studies. $V v G I D 2$ is a homology with rice F-box protein GID2 [19], which may be required for GAinduced degradation of DELLA via the proteasome pathway. In the present work, $V v G I D 2-1$ and $V v G I D 2-2$ may play important roles at GBS, and $V \nu G I D 2-2$ may also function at BRS. GBS and SHS are significant phases for GA biosynthesis, inactivation, and signal transduction of grape berry.

\section{Regulator genes in GA MST pathway during SBD}

GA signal can be positively or negatively modulated by many regulators like transcription factors and other regulatory genes. $V \nu G A M Y B$ [26], $V \nu S H I$ [27], and $V v P K L$ [28], which act downstream of DELLA, are identified as positively acting components in Arabidopsis. $V \nu G A M Y B, V \nu S H I$, and $V v P K L$ were expressed at low levels throughout the four stages in the present study. $V \nu S H I$ and $V \nu P K L$ play important roles in flowering regulatory network, leaf development, or repressing expression of embryonic traits but not in seed and fruit development $[27,28]$, whereas $V v G A M Y B$ is involved in GA-DELLA (VvSLR1)-VvmiR159c-VvGAMYB signal pathway, which modulate grapevine floral development and GA-induced grapevine parthenocarpy [2]. Similar to AtSPY [29], VvSPY2 acts as GA signaling repressor in grape berry as well. Additionally, $V v b H L H 137$ is a DELLA direct target [24] and possesses high expression levels in berries as well, indicating that VvbHLH137 may act as a crucial DELLA downstream target gene involved in SBD. Our results showed that $V v S P Y 1, V \nu S P Y 2$, and VvbHLH137 may be crucial regulatory factors in modulation of GA signal transduction during grape SBD.

Additionally, many GA-related miRNAs and their targets have been identified in orange [30] and Oryza sativa [1], which can facilitate the exploration of miRNA roles in GA MST pathway in grape berries. A functional interaction between miR159a and its target GAMYB in fruit development has been demonstrated in grape [29] and tomato [31]. Moreover, an increasing number of studies confirmed that miR319b [32], miR477 [33], and miR396 [34] are fruit development-related miRNAs, and miR159, miR319, miR477, and miR396 that responded to GA are also proven [35]. Overall, VvmiR159, VvmiR319, VvmiR477, and VvmiR396 may be induced by GA and involved in SBD. Therefore, transcription factor and miRNAs may contribute to maintain GA homoeostasis between GA synthesis and signal transduction process.

\section{Genes in multihormone MST pathways during SBD}

Auxin acts as a positive regulator during fruit set and growth and negative regulator during fruit maturation and ripening [36]. In the present study, IAA content was highest at SHS and decreased until BRS. GBS was a key phase for $V v Y U C 10-4$ and VvTAR4-1 involved in auxin biosynthesis, with auxin accumulation until SHS possibly 
reaching the highest level. Auxin produced by the seed in growing fruit acts to prevent ripening and premature dispersal before the seeds are fully developed [37]. Similarly, IAA content of berries decreased from SHS when seeds were fully developed. Furthermore, GBS was also an important phase for genes expressing during auxin signal transduction, such as $V v A R F 2-2$ and AUX/IAA protein. In tomato, auxin indirectly regulates ARF activity by promoting turnover of Aux/IAA proteins [38]. This promotion allows the ARFs to become active and impose their regulatory influence on the expression levels of auxin-responsive genes [38]. Thus, similar to tomato $[39,40]$, an ARF-IAA may interact to mediate auxin signaling and be directly involved in grape SBD. We indicated that VVSAUR may be associated with a new function in stimulating grape berry ripening. $V \nu S A U R 2$ especially showed high expression during BVS and BRS berry, which was different from ZmSAUR2 involving in auxin-mediated cell elongation in maize [41]. A rice OsSAUR39 gene negatively regulates auxin synthesis and transport [42]. VvSAUR2 was similar to OsSAUR9, whose expression pattern was opposite to active auxin level in berry. This phenomenon may be caused by a complex network for VvSAURs responding auxin in grape.

In previous study [43], ABA can be considered as a candidate ripening control factor in grape, but we considered that ABA may also be involved in regulating grape seed development. ABA is important during fruit development [44], ripening [45], and seed development [46]. Traditionally, $V v N C E D 1$ genes are expressed only at the beginning of ripening when $\mathrm{ABA}$ accumulation is high and $\mathrm{ABA}$ content is low in unripe fruit, increasing during fruit ripening [43]. Nevertheless, in the present study, a contradictory conclusion was drawn that ABA levels were much higher in green berries than in ripening berries (Fig. 1d). Similar to apple [44], ABA content also decreases from young fruit to older fruit and then increases during the ripening process in apple fruits. The highest ABA content in green berries was detected, implying that $\mathrm{ABA}$ may function as an indispensable hormone regulating seed development. Furthermore, $V v N C E D s$ were noted to be at a higher level at GBS. NCED gene encodes a key rate-limiting enzyme in ABA biosynthesis [43], and CYP707A is a key enzyme for ABA metabolism. Both were abundantly detected at GBS, but $V v N C E D s$ expression was significantly higher than $V v C Y P 707 A s$ expression, suggesting that $V v N C E D s$ played a stronger role than $V \nu C Y P 707 s$ at GBS. The only reasonable explanation is that $\mathrm{ABA}$ levels required for seed development are higher than those required for ripening process in grape. In Arabidopsis [47, 48], two peaks of ABA accumulation appear in the mid and late stages during seed development, but only one peak of
ABA level at GBS was observed in this study. This result may be caused by sampling times or different species. Furthermore, $V v P P 2 C 25-1$ and $V v P P 2 C 49$ may be the key factors of PP2C family in negatively regulating endogenous ABA content and response during grape SBD, especially at GBS.

Generally, cytokinin activities were found to peak shortly in young fruit progressing through the cell division phase, whereas activities were low or undetectable in ripening fruit. However, recent studies report of greatly increased cytokinin in the flesh of ripening kiwi fruit [49] and grapes [50], suggesting that cytokinin is also implicated in the control of ripening-related processes. In the present study, ZR levels reached two peaks at SHS, being active during cell division and being ripening phase at BRS. In grape [51], genes that regulated cytokinin biosynthesis (IPTs), activation (LOGs), and degradation (CKXs) are found to be expressed in four stages of berry development. Previous studies showed that VvIPTs are also found in other grape organs [51], in agreement with reports from Arabidopsis [52], tomato [53], and soybean [54]. The irreversible degradation of cytokinins by CKXs enzymes in grape berries is restricted to early developmental stages [24, 55]. Additionally, as previously reported $[24,55], V \nu C K X 5$ shows the highest expression at GBS and then exhibits progressive decrease of $V v C K X 5$ transcripts, which may contribute to the large increase in isopentenyladenine concentrations in post-veraison of grape berries $[56,57]$.

\section{Model of GA-mediated multihormone regulatory networks of SBD in grape}

Many studies related to one or two hormones regulating berry development have recently been reported [1, 53, 58, 59]. Nevertheless, few pertinent evidence are available on hormonal crosstalk during berry development in grape. A complex berry development regulatory network of GA downstream plant hormone signaling, and cross talk were demonstrated in this study to clarify the key nodes and nexuses in grape berries (Fig. 7). In the present study, biosynthesis enzymes GA20oxs andGA3oxs and GA receptor GID1B were highly expressed at GBS, meanwhile high expression levels of repressor DELLA proteins of GA MST pathway was also detected at this stage. Furthermore, GA3ox1, GA20ox2, and GID1Bmay be direct DELLA targets in Arabidopsis [24]. Thus, we supposed that a feedback mechanism existed as VvGAI1-4-VvGA20ox, VvGAI1-4-VvGA3ox, and $V v G A I 1-4-V v G I D 1 B$ to maintain the balance of GA content in regulating grape berry development. Furthermore, other hormones [60,61] influence GA levels by regulating $V v G A 20 o x s$ and $V \nu G A 3 o x s$ expression levels. Overall, GA20oxs, GA3oxs, and GA2oxs are not merely core acting enzymes in GA metabolism, but upstream 


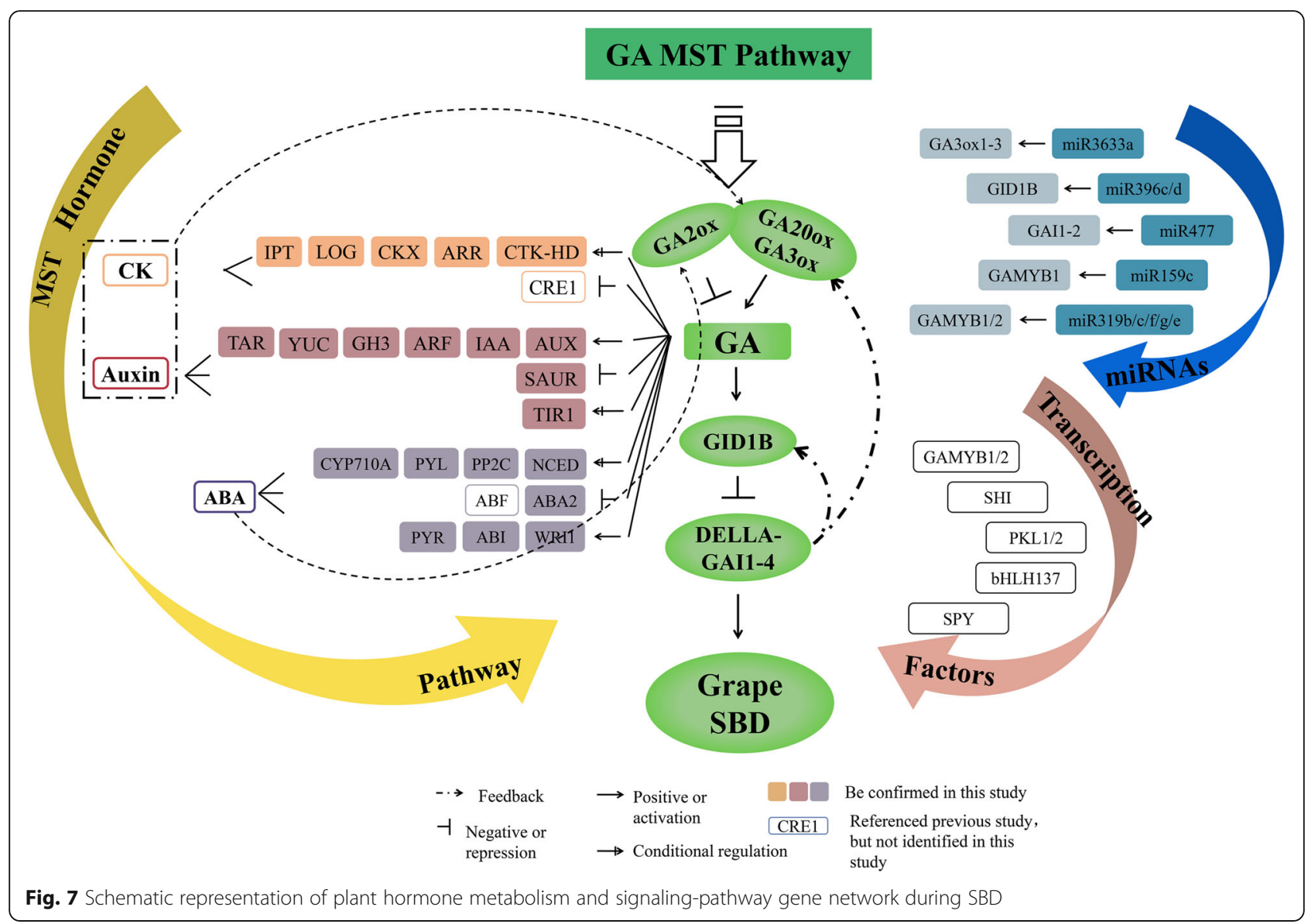

factors also respond to other hormones in GA MST pathway (Fig. 7). In addition, the crosstalk between GA and IAA [62], ABA [63], CK [61] in regulating SBD is also proved [63]. Many studies have demonstrated that bioactive GA controls plant growth and development, at least in part, by depressing the repressive effect of DELLA proteins [64]. Additionally, interactions as GA-auxin [58] and GA-CK [65] hormone signal are also mediated by DELLA proteins to control various processes in plant. GA may act downstream of auxin involved in early fruit development of Arabidopsis [66] and tomato [36]. Additionally, ABA also regulates tomato fruit set along with GA [66]. Different from the active ABA accumulation in the mid and late stages during seed development [43], GA biosynthesis appears to be more active in the early stage of seed development [67]. Thus, further functional studies are needed to validate the networks of GA and multihormone in grape SBD. This regulatory network could facilitate dissecting the molecular mechanisms underlying genes of other hormones MST pathway, respond to GA, and provide insights into the relationship between hormones and SBD in grape.

\section{Conclusion}

In the present study, total endogenous $\mathrm{GA}_{3}$ content significantly decreased during SBD, and a total of 48 related genes in GA M (31) and ST (17) pathway were identified by using transcriptome sequencing. Most genes of $V \nu G A 20 o x, V v G A 2 o x$, and $V v G A 3 o x$ family in GA M pathway expressed variously during SBD, yielding a metabolism flux toward GA biosynthesis. In GA $M$ pathway, out of 31 genes, $V v G A 200 \times 1-1, V v G A 3 o x 4-1$, and $V v G A 2 o x 1-1$ may be the major factors at GBS in interacting GA accumulation rate. Furthermore, $V v G A 200 \times 1-1$ and $V v G A 3 o x 4-1$ were more active than $V v G A 2 o x 1-1$, being consistent with the fact that GA was synthesized for seed development. The visible correlation between endogenous $\mathrm{GA}_{3}$ content and gene expression profiles suggested that transcriptional regulation of GA biosynthesis pathway genes is a key mechanism of GA accumulation at the SHS. We revealed feedback regulation as $V v G A 3 o x s-V v G A I 1-4$, $V \nu G A 2 o x s-V v G A I 1-4$, and $V v G I D 1 B-V v G A I 1-4$, maintaining the balance of $\mathrm{GA}_{3}$ content in berries. Moreover, 11 miRNAs may be involved in modulation of GA MST pathway by mediating their target genes, such as $V \nu G A 3 o x, \quad V v G I D 1 B$, and $V v G A M Y B$. Moreover, we 
found that crosstalk between GA and auxin, ABA and CK may modulate the complex process during SBD by the interaction network of genes of multihormone pathway. Lastly, based on the expression characterization of multihormone MST pathway genes, a putative schematic model of GA-mediated multihormone regulatory network during SBD was proposed, which may provide novel insight to explore GA-mediated regulatory networks during SBD in grape. Our results may provide novel insight to explore GA-mediated regulatory networks during SBD in grape.

\section{Methods}

\section{Plant material}

Three-year-old 'Fujiminori' grape trees grown in Nan-

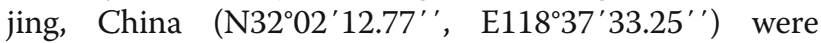
chosen as the experimental material. The plant materials were grown under common field conditions at the Jiangsu Vocational College of Agriculture and Forestry grape farm, Jurong, China (our long-term partners). Berry samples were collected at different stages: green berry stage (GBS) [10 days after flowering (10 DAF)], stone-hardening stage (SHS) (40 DAF), berry veraison stage (BVS) (65 DAF) and berry ripening stage (BRS) (90 DAF) throughout the growing season. A total of 12 samples, comprising four stages berry, and including three biological replicates, were sequenced. RNA for Illumina sequencing was purified from 40 uniform and averagesized berries with seeds sampled from 20 clusters to study a representative biological selection of transcripts at each stage.

Total RNA extraction, construction of cDNA library, and Illumina deep sequencing

Total RNA of different stages (i.e., GBS, SHS, BVS, and BRS) berry samples was extracted using TRIzol reagent (Invitrogen, Carlsbad, CA, USA). Sequencing libraries were generated using NEBNext ${ }^{\circ}$ UltraTM RNA Library Prep Kit for Illumina ${ }^{\circ}$ (NEB, USA) following manufacturer's recommendations. The transcriptome were sequenced using Illumina $\mathrm{HiSeq}^{\mathrm{Tm}} 2500$ Shanghai Hanyu Biotechnology Company (Shanghai, China) [68].

\section{Accession code}

The RNA-seq data have been deposited into the NCBI under the accession number SRP068911 (https://www. ncbi.nlm.nih.gov/sra?term=SRP068911) and GSE153169 (https://www.ncbi.nlm.nih.gov/geo/query/acc.cgi?acc= GSE153169).

\section{Identification and analysis of the GA MST and multihormone MST pathway genes}

GA MST pathway and multihormone MST pathway genes were identified via BLAST $\mathrm{P}$ search from NCBI using Arabidopsis gene sequences as queries. We gained the grape genome and a set of annotated gene sequences from Grape genome data Get Data: Grapevine V1 Annotation. And a survey was conducted to affirm these genes by functional annotation from grape transcriptome and confirm the reliability of the initial results (RNA-Seq data accession SRP068911) [69].

\section{Morphological, physiological, and biochemical variations during SBD}

The gene location in the chromosome of each GA MST pathway genes was obtained from the grape genome (http://www.genoscope.cns.fr/externe/ Genome Browser/Vitis/). As Leng [69] described, gene duplication events was detected using MCScanX software (http://chibba.pgml.uga.edu/mcscan2/). We used protein sequences to construct phylogenetic trees in MEGA 6.0 software using the neighbor-joining method with 1000 bootstrap replicates [70], and intron/exon structures were predicted using the Gene Structure Display Server (http://gsds.cbi.pku.edu.cn/index.php), and conserved domains were searched using SMART (http://smart. embl-heidelberg.de/). Motifs were searched using the MEME 5.05 online program (http://meme-suite.org/ tools/meme). The optimized parameters of MEME were employed as follows: number of repetitions, any; maximum number of motifs, 15; and the optimum width of each motif, between 6 and 50 residues.

\section{Examination of endogenous $G_{3}, I A A, Z R$, and $A B A$}

Berries of four stages, G, SH, V, and R, were chosen. Endogenous $\mathrm{GA}_{3}$, IAA, $\mathrm{ZR}$, and ABA levels were determined by using an enzyme-linked immunosorbent assay kit (ELISA) (Rapidbio, USA), according to the manufacturer's instructions and as described in previous study [71]. Each berry with seed was sampled from 40 berries of 20 clusters as replicates.

\section{Expression analysis of genes of GA and multihormone MST pathway}

Differential expression was analyzed and calculated using the edge $R$ packkage [72]. If transcripts with "FDR $<0.001$ " and " $\mid \log 2$ fold-change $(|\log 2 \mathrm{FC}|) \geq 1$ ", they will be identified as significant differences in transcription level. And transcripts with $|\log 2 \mathrm{FC}|<0.25$ were supposed to have no change, while transcripts with $0.25<|\log 2 \mathrm{FC}|<1$ were considered as "changed slightly" in expression level [69].

\section{RT-qPCR validation in different grape varieties}

RT-qPCR was used to verify the expression patterns of GA MST pathway genes from RNA-Seq. Total RNA samples of four stages (i.e., 10, 20, 30, and 50 DAA) of Vitis vinifera $\times V$. labrusca ('Jingya' and 'Izunishiki') and 
V. viniferal. ('Queenora Seedless' and 'Madeleine Angenine') were extracted using our modified cetyltrimethyl ammonium bromide (CTAB) method [73]. Purified RNA samples were reverse transcribed using the PrimeScript RT Reagent Kit with gDNA Eraser (Takara, Dalian, China). Gene specific primers were designed using Primer3 software (http://primer3.ut.ee/) (Additional file 4: Table S4). For each reaction, a total volume of $20 \mu \mathrm{l}$ contained $10 \mu \mathrm{l}$ of $2 \times$ SYBR green reaction mix, $2.0 \mu \mathrm{l}$ of diluted cDNA, and $0.2 \mu \mathrm{M}$ of each primer. And three replicates of each cDNA sample were performed and normalized according to the internal control. The relative gene expression data were analyzed using the $2^{-\Delta \Delta C T}$ method [74].

\section{Supplementary information}

Supplementary information accompanies this paper at https://doi.org/10. 1186/s12870-020-02591-1.

Additional file 1: Table S1. The gene expression patterns of GA MST pathway genes in four stages of grape SBD.

Additional file 2: Table S2. The gene expression patterns of auxin, $A B A$ and CK MST pathway genes in three stages of grapevine SBD.

Additional file 3: Table S3. 64 genes expression patterns of GA, auxin, ABA and CK MST pathway in seed, berry skin, pericarp and flesh from a global transcriptomic atlas.

Additional file 4: Table S4. The primers sequences of GA MST pathway genes for $q R T-P C R$.

Additional file 5: Table S5. The name, stage and type of data used in this study.

\section{Abbreviations}

ABA: Abscisic acid; CK: Cytokinin; AUX/IAA: Auxin-response repressor protein/ Indole Acetic Acid; ARF: Auxin response factor; CKX: Cytokinin dehydrogenase; GA20x: Gibberellin 2-oxidase; GA20ox: Gibberellin 20oxidase; GA3ox: Gibberellin 3-oxidase; GID1: Gibberellin insensitive dwarf1; NCED: 9-cis-epoxycarotenoid dioxygenase; PKL: PICKLE; PP2C: Protein phosphatase 2C; SAUR: Small auxin-upregualted RNAs; SHI: Short internodes; SPY: Spindly; TAR: Tryptophan aminotransferase; YUC: Yucca

\section{Acknowledgements}

The authors are grateful for the research laboratory facilities provided by the College of Horticulture, Nanjing Agricultural University, Nanjing, China.

\section{Authors' contributions}

WW and CW conceived and designed the research. WW and YB conducted experiments. LG and $Y Z$ contributed powerful analytical tools. WW, XW, ZL and $\mathrm{HJ}$ analyzed data, and WW and CW wrote the manuscript. KP and JF revised manuscript. All authors read and approved the final manuscript.

\section{Funding}

The authors are grateful for the research laboratory facilities provided by the College of Horticulture, Nanjing Agricultural University, Nanjing, China. This work was supported by the National Key Research and Development Program of China (2018YFD1000106), Natural Science Foundation of Jiangsu Province (BK20181318) and National Natural Science Foundation of China (31972373, 31801809). Founding body had no role in the design of the study and collection, analysis, and interpretation of data and in writing the manuscript.

\section{Availability of data and materials}

The RNA-seq data have been deposited into the NCBI under the accession number SRP068911 (https://www.ncbi.n/m.nih.gov/sra?term=SRP068911) and GSE153169 (https://www.ncbi.nlm.nih.gov/geo/query/acc.cgi?acc=GSE15316
9). All data generated or analyzed during this study are included in this published article and its additional files.

\section{Ethics approval and consent to participate}

The plants used in our study are not endangered species. Plant sample collection was performed in accordance with the local legislation in China.

\section{Consent for publication}

Not applicable.

\section{Competing interests}

The authors declare that they have no competing interests.

\section{Author details}

${ }^{1}$ Nanjing Agricultural University, College of Horticulture, Nanjing 210095, PR China. ${ }^{2}$ China Agricultural University, College of Horticulture, Beijing 100193, China. ${ }^{3}$ Division of Crop Sciences, ICAR-Central Research Institute for Dryland Agriculture, Santoshnagar, Hyderabad, Telangana 500059, India. ${ }^{4}$ Institute of Pomology, Jiangsu Academy of Agricultural Sciences, Nanjing 210014, China.

Received: 23 January 2020 Accepted: 12 August 2020

Published online: 21 August 2020

References

1. Tang YY, Liu HH, Guo SY, Wang B, Li ZT, Chong K, Xu YY. OsmiR396d miRNA affects gibberellin and brassinosteroid signaling to regulate plant architecture. Plant Physiol. 2018;176:946-59.

2. Wang C, Sudisha J, Zhang WY, Abdelrahman M, Fang JG. Spatio-temporal expression of miRNA159 family members and their GAMYB target gene during the modulation of gibberellin-induced grapevine parthenocarpy. J Exp Bot. 2018;69:3639-50.

3. Cheng C, Xu XZ, Singer SD, Li J, Zhang HJ, Gao M, Wang L, Song JY, Wang $X P$. Effect of GA3 treatment on seed development and seed related gene expression in grape. PLoS One. 2013;8:e80044.

4. Zhang C, Tanabe K, Tamura F, Itai A, Yoshida M. Rolesof gibberellins in increasing sink demand in Japanese pear fruit during rapid fruit growth. Plant Growth Regul. 2007:52(2):161-72.

5. Chen JJ, Xie JH, Duan YJ, Hu HG, Li WM. Genome-wide identification and expression profiling reveal tissue-specific expression and differentiallyregulated genes involved in gibberellin metabolism between Williams banana and its dwarf mutant. BMC Plant Biol. 2016;16:123.

6. Zhao HJ, Dong J, Wang T. Function and expression analysis of gibberellin oxidases in apple. Plant Mol Biol Report. 2010;28:231-8.

7. Reid JB, Davidson SE, Ross JJ. Auxin acts independently of DELLA proteins in regulating gibberellin levels. Plant Signal Behav. 2011;6:406-8.

8. Luo DL, Ba LJ, Shan W, Kuang JF, Lu WJ, Chen JY. Involvement of WRKY transcription factors in ABA-induced cold tolerance of Banana fruit. J Agric Food Chem. 2017:65:3627-2635.

9. Fasoli M, Dal Santo S, Zenoni S, Battista Tornielli G, Farina L, Zamboni A, Porceddu A, Venturini L, Bicego M, Murino V, Ferrarini A, Delledonne M, Pezzotti M. The grapevine expression atlas reveals a deep Transcriptome shift driving the entire plant into a maturation program. Plant Cell. 2012;24: 3489-505.

10. De JM, Mariani C, Vriezen WH. The role of auxin and gibberellin in tomato fruit set. J Exp Bot. 2009;60:1523-32.

11. Csukasi F, Osorio S, Gutierrez JR, Kitamura J, Giavalisco P, Nakajima M, Fernie AR, Rathjen JP, Botella MA, Valpuesta V, Medina-Escobar N. Gibberellin biosynthesis and signalling during development of the strawberry receptacle. New Phytol. 2011:191:376-90.

12. Tsaballa A, Pasentsis K, Tsaftaris AS. The role of a Gibberellin 20-Oxidase gene in fruit development in pepper (Capsicum annuum). Plant Mol Biol Report. 2012;30:556-65.

13. Ozga JA, Yu J, Reinecke DM. Pollination-, development-, and auxin-specific regulation of gibberellin $3 \beta$-hydroxylase gene expression in pea fruit and seeds. Plant Physiol. 2003;131:1137-46.

14. Rebers M, Kaneta T, Kawaide $H$, Yamaguchi S, Yang YY, Imai R, Sekimoto $H$, KAmiya Y. Regulation of gibberellin biosynthesis genes during flower and early fruit development of tomato. Plant J. 1999;17:241-50.

15. Fuentes S, Ljung K, Sorefan K, Alvey E, Harberd NP, Østergaard L. Fruit growth in Arabidopsis occurs via DELLA-dependent and DELLA-independent gibberellin responses. Plant Cell. 2012;24:3982-96. 
16. Chai LJ, Li YM, Chen SW, Perl A, Zhao FX, Ma HQ. RNA sequencing reveals high resolution expression change of major plant hormone pathway genes after young seedless grape berries treated with gibberellin. Plant Sci. 2014; 229:215-24.

17. Guo CL, Guo RR, Xu XZ, Gao M, Li XQ, Song JY, Zheng Y, Wang XP. Evolution and expression analysis of the grape (Vitis vinifera L.) WRKY gene family. J Exp Bot. 2014;65:1513-28.

18. Holub EB. The arms race is ncient history in Arabidopsis, the wildflower. Nat Rev Genet. 2001;2(7):516-27.

19. Sasaki A, Itoh H, Gomi K, Ueguchi-Tanaka M, Ishiyama K, Kobayashi M, Jeong $\mathrm{DH}, \mathrm{An} \mathrm{G}$, Kitano H, Ashikari M, Matsuoka M. Accumulation of phosphorylated repressor for gibberellin Signaling in an F-box mutant. Science. 2003:299:1896-8.

20. Boonkaew T, Mongkolsiriwatana C, Vongvanrungruang A, Srikulnath K, Peyachoknagul S. Characterization of GA20ox genes in tall and dwarf types coconut (Cocos nucifera L.). Genes Genomics. 2018;40:735-45.

21. Du Q, Li CL, Li DQ, Lu SF. Genome-wide analysis, molecular cloning and expression profiling reveal tissue specifically expressed, feedback-regulated, stress-responsive and alternatively spliced novel genes involved in gibberellin metabolism in Salvia miltiorrhiza. BMC Genomics. 2015;16:1087.

22. Rasori A, Ziliotto F, Botton A, Bonghi C, Ramina A. Hormonal cross talk between fruit and seed throughout development and ripening in peach. Acta Hortic. 2010;884:53-60.

23. Shiozaki S, Ogata T, Horiuchi S. Endogenous polyamines in the pericarp and seed of the grape berry during development and ripening. Sci Hortic. 2000; 83:33-41.

24. Zentella R, Zhang ZL, Park M, Thomas SG, Endo A, Murase K, Fleet CM, Jikumaru Y, Nambara E, Kamiya Y, Sun TP. Global analysis of DELLA direct targets in early gibberellin signaling in Arabidopsis. Plant Cell. 2007:19:3037-57.

25. Deluc LG, Grimplet J, Wheatley MD, Tillett RL, Quilici DR, Osborne C, Schooley DA, Schlauch KA, Cushman JC, Cramer GR. Transcriptomic and metabolite analyses of cabernet sauvignon grape berry development. BMC Genomics. 2007;8:429.

26. Achard P, Herr A, Baulcombe DC, Harberd NP. Modulation of floral development by a gibberellin-regulated microRNA. Development. 2004;131: 3357-65.

27. Baylis T, Cierlik I, Sundberg E, Mattsson J. SHORT INTERNODES/STYLISH genes, regulators of auxin biosynthesis, are involved in leaf vein development in Arabidopsis thaliana. New Phytol. 2013;197:737-50.

28. Henderson JT, Li HC, Rider SD, Mordhorst AP, Romero-Severson J, Cheng JC, Robey J, Sung RZ, Vries SCD, Ogas J. PICKLE acts throughout the plant to repress expression of embryonic traits and may play a role in gibberellindependent responses. Plant Physiol. 2004;134:995-1005.

29. Jacobsen SE, Olszewski NE. Mutations at the SPINDLY locus of Arabidopsis alter gibberellin signal transduction. Plant Cell. 1993;5:887-96.

30. Liu YL, Wang L, Chen DJ, Wu XM, Huang D, Chen LL, Li L, Deng XX, Xu Q. Genome-wide comparison of microRNAs and their targeted transcripts among leaf, flower and fruit of sweet orange. BMC Genomics. 2014;15:695.

31. Silva EMD, Silva GFFE, Bidoia DB, Azevedo MDS, Jesus FAD, Pino LE, Peres LEP, Carrera E, López-Díaz I, Nogueira FTS. microRNA159-targeted SIGAMYB transcription factors are required for fruit set in tomato. Plant J. 2017:92:95109.

32. Saminathan $T$, Bodunrin A, Singh NV, Devarajan R, Nimmakayala P, Jeff M, Aradhya M, Reddy UK. Genome-wide identification of microRNAs in pomegranate (Punica granatum L.) by high-throughput sequencing. BMC Plant Biol. 2016;16:122

33. Liu M, Pirrello J, Chervin C, Roustan JP, Bouzayen M. Ethylene control of fruit ripening: revisiting the complex network of transcriptional regulation. Plant Physiol. 2015;169:2380-90.

34. Leng XP, Fang JX, Pervaiz T, Ii Y, Wang XM, Liu D, Zhu XD, Fang JG. Characterization of expression patterns of grapevine microRNA family members using microRNA rapid amplification of complementary DNA ends. Plant Genome. 2015;8(2):1-9.

35. Han J, Fang JG, Wang C, Yin YL, Sun X, Leng XP, Song CN. Grapevine microRNAs responsive to exogenous gibberellin. BMC Genomics. 2014; $15: 111$.

36. Pattison RJ, Csukasi F, Catalá C. Mechanisms regulating auxin action during fruit development. Physiol Plant. 2014;151:62-72.

37. Sundberg E, Østergaard L. Distinct and dynamic auxin activities during reproductive development. Cold Spring Harb Perspect Biol. 2009;1:a001628.
38. Goetz M, Vivian-Smith A, Johnson SD, Koltunow AM. AUXIN RESPONSE FACTOR8 is a negative regulator of fruit initiation in Arabidopsis. Plant Cell. 2006;18:1873-86.

39. Bassa C, Mila I, Bouzayen M, Audran-Delalande C. Phenotypes associated with down-regulation of SI-IAA27 support functional diversity among auX/ IAA family members in tomato. Plant Cell Physiol. 2012;53:1583-95.

40. Hendelman A, Buxdorf K, Stav R, Kravchik M, Arazi T. Inhibition of lamina outgrowth following Solanum lycopersicum AUXIN RESPONSE FACTOR 10 (SIARF10) derepression. Plant Mol Biol. 2012;78:561-76.

41. Knauss $S$, Rohrmeier $T$, Lehle $L$. The auxin-induced maize gene ZmSAUR2 encodes a short-lived nuclear protein expressed in elongating tissues. J Biol Chem. 2003;278:23936-43.

42. Kant S, Rothstein S. Auxin-responsive SAUR39 gene modulates auxin level in rice. Plant Signal Behav. 2009:4:1174-5.

43. Zhang M, Leng P, Zhang GL, Li XX. Cloning and functional analysis of 9-cisepoxycarotenoid dioxygenase (NCED) genes encoding a key enzyme during abscisic acid biosynthesis from peach and grape fruits. J Plant Physiol. 2009; 166:1241-52.

44. Bcrutci J. Effect of abscisic acid on sorbitol uptake in growing apple fruits. J Exp Bot. 1983;34:737-43.

45. Nakashima K, Yamaguchi-Shinozaki K. ABA signaling in stress-response and seed development. Plant Cell Rep. 2013;32:959-70.

46. Finkelstein RR, Lynch TJ. The Arabidopsis Abscisic acid response gene AB/5 encodes a basic Leucine zipper transcription factor. Plant Cell. 2000;12:599609.

47. Koornneef M, Hanhart CJ, Hilhorst HWM, Karssen CM. In vivo inhibition of seed development and reserve protein accumulation in recombinants of abscisic acid biosynthesis and responsiveness mutants in Arabidopsis thaliana. Plant Physiol. 1989;90:463-9.

48. Karssen CM, Brinkhorstvanderswan DLC, Breekland AE, Koornneef M. Induction of dormancy during seed development by endogenous abscisic acid: studies on abscisic acid deficient genotypes of Arabidopsis thaliana $(\mathrm{L})$ Heynh. Planta. 1983;157:158-65.

49. Pilkington SM, Montefiori M, Galer AL, Emery RJN, Allan AC, Jameson PE. Endogenous cytokinin in developing kiwifruit is implicated in maintaining fruit flesh chlorophyll levels. Ann Bot. 2013;112:57-68.

50. Böttcher C, Boss PK, Davies C. Increase in cytokinin levels during ripening in developing Vitis vinifera cv. Shiraz berries. Am J Enol Vitic. 2013;64:527-31.

51. Böttcher C, Burbidge CA, Boss PK, Davies C. Changes in transcription of cytokinin metabolism and signalling genes in grape (Vitis vinifera L.) berries are associated with the ripening-related increase in isopentenyladenine. BMC Plant Biol. 2015;15:223.

52. Miyawaki K, Matsumoto-Kitano M, Kakimoto T. Expression of cytokinin biosynthetic isopentenyltransferase genes in Arabidopsis: tissue specificity and regulation by auxin, cytokinin, and nitrate. Plant J. 2004;37:128-38.

53. Matsuo S, Kikuchi K, Fukuda M, Honda I, Imanishi S. Roles and regulation of cytokinins in tomato fruit development. J Exp Bot. 2012;63:5569-79.

54. Le DT, Nishiyama R, Watanabe Y, Vankova R, Tanaka M, Seki M, Ham LH, Yamaguchi-Shinozaki K, Shinozaki K, Phan Tran LS. Identification and expression analysis of cytokinin metabolic genes in soybean under normal and drought conditions in relation to cytokinin levels. PLoS One 2012;7: e42411.

55. Pilati $S$, Perazzolli $M$, Malossini $A$, Cestaro $A$, Demattè $L$, Fontana $P, R i$, Viola R, Velasco R, Moser C. Genome-wide transcriptional analysis of grapevine berry ripening reveals a set of genes similarly modulated during three seasons and the occurrence of an oxidative burst at vèraison. BMC Genomics. 2007:8:428.

56. Galuszka P, Popelková H, Werner T, Frébortová J, Pospíšilová H, Mik V, Köllmer I, Schmülling T, Frébort I. Biochemical characterization of cytokinin oxidases/dehydrogenases from Arabidopsis thaliana expressed in Nicotiana tabacum L. Plant Growth Regul. 2007;26:255-67.

57. Bilyeu KD, Cole JL, Laskey JG, Riekhof WR, Esparza TJ. Molecular and biochemical characterization of a cytokinin oxidase from maize. Plant Physiol. 2001;125:378-86.

58. Hu JH, Israeli A, Ori N, Sun TP. DELLA-ARF/IAA interaction mediates crosstalk between gibberellin and Auxin Signaling in controlling fruit initiation in Solanum lycopersicum. Plant Cell. 2018;30:1710-28.

59. Leclercq J, Ranty B, Sanchez-Ballesta MT, Li ZG, Jones B, Jauneau A, Pech JC, Latché A, Ranjeva R, Bouzayen M. Molecular and biochemical characterization of LeCRK1, a ripening-associated tomato CDPK-related kinase. J Exp Bot. 2005;56:25-35. 
60. Srivastava MK, Dwivedi UN. Delayed ripening of banana fruit by salicylic acid. Plant Sci. 2000;158:87-96.

61. Lu L, Liang JJ, Zhu X, Xiao K, Li TZ, Hu JF. Auxin- and cytokinin-induced berries set in grapevine partly rely on enhanced gibberellin biosynthesis. Tree Genet Genomes. 2016;12:41.

62. Shinozaki Y, Hao SH, Kojjima M, Sakakibara H, Ozeki-lida Y, Zheng Y, Fei ZJ, Zhong SL, Giovannon JJ, Rose JKC, Okabe Y, Heta Y, Ezura H, Ariizumi T. Ethylene suppresses tomato (Solanum lycopersicum) fruit set through modification of gibberellin metabolism. Plant J. 2015;83:237-51.

63. Murcia G, Pontin M, Piccoli P. Role of ABA and Gibberellin A3 on gene expression pattern of sugar transporters and invertases in Vitis vinifera $\mathrm{Cv}$. Malbec during berry ripening. Plant Growth Regul. 2017;84:275-83.

64. Richards DE, King KE, Ait-Ali T, Harbersd NP. How gibberennlin regulates plant growth and development: a molecular genetic analysis of gibberellin signalling. Annu Rev Plant Physiol Plant Mol Biol. 2001;52:67-88.

65. Achard P, Baghour M, Chapple A, Hedden P, Straeten DVD. The plant stress hormone ethylene controls floral transition via DELLA-dependent regulation of floral meristem-identity genes. Proc Natl Acad Sci. 2007;104:6484-9.

66. Dorcey E, Urbez C, Blázquez AM, Carbonell J, Perez-Amador MA.

Fertilization-dependent auxin response in ovules triggers fruit development through the modulation of gibberellin metabolism in Arabidopsis. Plant J. 2009:58:318-32.

67. Kim YC, Nakajima M, Nakayama A, Yamaguchi I. Contribution of gibberellins to the formation of Arabidopsis seed coat through starch degradation. Plant Cell Physiol. 2005;46:1317-25.

68. Shangguan Lf MQ, Fang X, Zhang KK, Jia HF, Li XP, Bao YQ, Fang JG. RNAsequencing reveals biological networks during table grapevine ('Fujiminori') fruit development. PLoS One. 2017;12(1):e0170571.

69. Leng XP, Wang PP, Wang C, Zhu XD, Li XP, Li HY, Mu Q, Li A, Liu ZJ, Fang $J G$. Genome-wide identification and characterization of genes involved in carotenoid metabolic in three stages of grapevine fruit development. Sci Rep. 2017;7:4216.

70. Tamura K, Stecher C, Peterson D, Filipski A, Kumar S. MEGA6: molecular evolutionary genetics analysis version 6.0. Mol Biol Evol. 2013;30:2725-9.

71. Yang JF, Guo ZF. Cloning of a 9-cis-epoxycarotenoid dioxygenase gene (SgNCED 1) from Stylosanthes guianensisand its expression in response to abiotic stresses. Plant Cell Rep. 2007;26:1383-90.

72. Robinson MD, McCarthy DJ, Smyth GK. edgeR: a bioconductor package for differential expression analysis of digital gene expression data. Bioinformatics. 2009;26:139-40.

73. Wang C, Wang XC, Kibet NK, Song CN, Zhang CQ, Li XY, Han J, Fang JG. Deep sequencing of grapevine flower and berry short RNA library for discovery of novel microRNAs and validation of precise sequences of grapevine microRNAs deposited in miRBase. Physiol Plant. 2011;143:64-81.

74. Livak KJ, Schmittgen TD. Analysis of relative gene expression data using realtime quantitative PCR and the 2- $\Delta \Delta C T$ method. Methods. 2001;25:402-8.

\section{Publisher's Note}

Springer Nature remains neutral with regard to jurisdictional claims in published maps and institutional affiliations.

\section{Ready to submit your research? Choose BMC and benefit from:}

- fast, convenient online submission

- thorough peer review by experienced researchers in your field

- rapid publication on acceptance

- support for research data, including large and complex data types

- gold Open Access which fosters wider collaboration and increased citations

- maximum visibility for your research: over $100 \mathrm{M}$ website views per year

At $\mathrm{BMC}$, research is always in progress.

Learn more biomedcentral.com/submissions 